\title{
Nutrition in acute surgical patients - multi disciplinary team approach to increasing uptake of the malnutrition universal screening tool
}

\author{
N. Sriram ${ }^{1}$ and A. Tangri ${ }^{2}$ \\ ${ }^{1}$ John Radcliffe Hospital, Headington OX3 9DU, UK and ${ }^{2}$ Stoke Mandeville Hospital, Aylesbury HP21 8AL, UK
}

\begin{abstract}
Malnutrition is a significant health burden of the acute care admission, with prevalence varying from $13-46 \%$ in several studies ${ }^{(1)}$. The economic and healthcare implications of malnutrition are well recognized. Malnutrition is associated with increased in hospital stay and mortality (hospital, 3 and 6 month) ${ }^{(2)}$. Additionally, malnourished patients are at increased risk of impaired wound healing and electrolyte imbalance. In recognition of this issue, NICE (National Institute for Health and Clinical Excellence) has issued guidance regarding nutritional support in adults in $2007^{(3)}$. It advocates the use of well validated screening tools, such as the MUST (Malnutrition Universal Screening Tool) questionnaire, to identify malnutrition in patients on admission. However, there are few studies reflecting actual usage of the questionnaire in daily practice. For example, one published audit showed $79 \%$ of patients were screened, with only $39 \%$ screened at 24 hours $^{(4)}$.

A study was conducted in an acute surgical ward, auditing the use of the MUST questionnaire on admission. Over a 2 month period, the percentage of patients with BMIs (body mass index) and MUST scores documented was noted. The number of patients started on supplementation as a result, was also recorded. After the first round of audit, simple interventions focusing of the multi disciplinary team approach to increasing uptake of the MUST questionnaire were implemented. The results of the audit was presented to the medical team as well as the nursing staff with discussions to raise awareness. A reaudit was conducted showing significant improvement $(p<0.05$, Chi squared test) in measurement of the MUST score (refer to table 1).
\end{abstract}

\begin{tabular}{lcc}
\hline & Pre Intervention & Post Intervetnion \\
\hline MUST scores & 18 & 25 \\
Nil MUST scores & 32 & 10 \\
\hline
\end{tabular}

In conclusion, use of MUST questionnaires is an important initial screening tool for malnutrition on admission. However, a multi disciplinary team involving nurses and medical staff must take responsibility for MUST completion. Poor uptake of the questionnaire can hamper appropriate supplementation. From this audit, simple interventions involving different members of the team can improve outcomes. By increasing uptake of the questionnaire, further data can be gathered regarding the implications of regular nutritional screening.

1. Stratton RJ, Hackston A, Longmore D, Dixon R, Price S, Stroud M et al. Malnutrition in hospital outpatients and inpatients: prevalence, concurrent validity and ease of use of the 'malnutrition universal screening tool' ('MUST') for adults. Br J Nutr. 2004; 92(5), 799-808.

2. Stratton RJ, King CL, Stroud MA, Jackson AA \& Elia M 'Malnutrition Universal Screening Tool' predicts mortality and length of hospital stay in acutely ill elderly. Br J Nutr. 2006; 95(2), 325-30.

3. NICE guidelines: CG32 Nutrition support in Adults, http://guidance.nice.org.uk/CG32/Guidance/pdf/English

4. Barthorpe E, McLaughlin J, Kuczynska B \& Farrer K Uptake of the MUST screening tool for inpatient in a teaching hospital: an audit of progress to date. Malnutrition matters. 2008; 67, E127. 